\title{
Evaluation of Newer Insecticidal Formulation against Sucking Pests and Effect on Yield of Soybean (Glycine max L.)
}

\author{
Dinesh M. Chaudhary ${ }^{1 *}$, Mahesh M. Chaudhary ${ }^{2}$ and F.K. Chaudhary ${ }^{1}$ \\ ${ }^{1}$ Department of Agriculture Entomology, ${ }^{2}$ Department of Plant Pathology, C. P. College of \\ Agriculture, SDAU, S. K. Nagar-385 506, India \\ *Corresponding author
}

A B S T R A C T

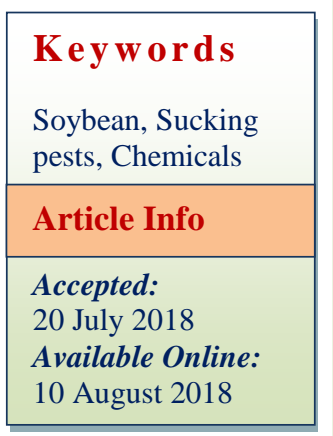

The investigations were carried out on management of sucking pests of soybean (Glycine $\max$ L.) at Agronomy Instructional Farm, Chimanbhai Patel College of Agriculture, Sardarkrushinagar Dantiwada Agricultural University, Sardarkrushi nagar during kharif, 2016. Among various chemical and non-chemical insecticides tested against sucking pest of soybean, imidacloprid 17.8 SL@0.005\% found effective against jassid and thrips while, acetamiprid 20 SP $0.004 \%$ was effective against whitefly in soybean. The highest grain yield of soybean recorded in the treatment of imidacloprid 17.8 SL @ $0.005 \%$ (1166 $\mathrm{kg} / \mathrm{ha}$ ). The net Protection Cost Benefit Ratio (PCBR) was highest in the treatment of imidacloprid (1: 23.67) followed by acetamiprid (1: 22.63), thiamethoxam (1: 17.06), dimethoate (1: 12.53) and neem oil $1500 \mathrm{ppm}$ (1: 05.29). The lowest avoidable loss was recorded in the plot treated with imidacloprid 17.8 SL followed by acetamiprid 20 SP $(4.45 \%)$ and thiamethoxam $25 \mathrm{WG}(6.34 \%)$. On other hand highest percentage of avoidable loss in soybean yield was observed in the untreated plot (32.16).

\section{Introduction}

Soybean (Glycine max L.) is one of the most important oil seed crop in the country grown for oil and protein production in both the rabi and kharif seasons. Seed contains about 42 per cent protein and 20 per cent oil providing 60 per cent of the world supply of vegetable protein and 30 per cent of the edible oil (Biswas, 2013).

For vegetarians, it is known as "poor man's meat." It is known as the "GOLDEN BEAN" of the $20^{\text {th }}$ Century. Though, Soybean is a legume crop. This crop suffers a lot due to the attack of number of insect pests (Lal et al., 1981). It is mainly attacked by gram pod borer, leaf eating caterpillar, green semilooper, grey semilooper, leaf miner, whitefly, stem fly, thrip, aphid, and jassid, (Ahirwar et al., 2015). The conventional method may not serve the need of sustainable and desirable insect pest control. Therefore, integration of insecticides with bio-pesticides may provide economic and effective management of the pests in soybean. 
Materials and Methods

To evaluate the efficacy of different insecticides against sucking pests on soybean, field trial was conducted at Agronomy Instructional Farm, C. P. College of Agriculture, S. D. Agricultural University, Sardarkrushinagar during kharif, 2016.

\section{Application of insecticides}

All the insecticides were applied as foliar spray with the help of knapsack sprayer fitted with hollow cone nozzle.

The sprayer was washed thoroughly prior to the application of subsequent treatments and second spray was given after fifteen days of first spray.

\section{Method of recording observations}

Observations on number of jassid, whitefly and thrips were recorded on five randomly selected plants from each treatment before and after 3, 7 and 10 days of spraying from three leaves (top, middle and bottom).

The data thus obtained were statistically analysed.

\section{Method of evaluation}

Per cent increase in yield over control and avoidable loss

At harvest the grain yield was recorded separately for each treatment. On the basis of yield the economics was calculated. Increase in yield over control and avoidable loss were calculated applying formula given by Khosla (1977).

Yield in treatment - Yield in control Increase in yield over control $(\%)=-----\mathrm{x} 100$

Yield in control
Highest yield in treated plot Yield in treatment Avoidable loss $(\%)=$ $\mathrm{x} 100$

Highest yield in treated plot

\section{Results and Discussion}

With a view to find out the efficacy of different chemical as well as non-chemical insecticides against sucking pests (jassid, whitefly and thrips) in soybean.

\section{Jassid (E. kerri)}

The results on jassid incidence per three leaves before and after spraying are summarized in Table 1. Based on results of first and second spray, it was clearly observed that imidacloprid 17.8 SL @ $0.005 \%$ was the most effective treatment in controlling jassids under field conditions followed by acetamiprid 20 SP @ $0.004 \%$ and dimethoate 30 EC@ $0.03 \%$.

The treatments of thiamethoxam 25 WG, buprofezin $25 \mathrm{SC}$ and chlorfenapyr $10 \mathrm{SC}$ formed the next group of effective insecticides. Similarly botanical insecticide (neem oil 1500 ppm @ $0.5 \%$ ) and biopesticides (Beauveria bassiana $2 \times 10^{8} \mathrm{cfu} / \mathrm{g}$ and Lecanicillium lecanii $2 \times 10^{8} \mathrm{cfu} / \mathrm{g}$ ) have also proved effective against untreated control. Looking to the safety point of view all the non-chemical pesticides can be incorporated in IPM programmes against jassid management in soybean.

Joshi and Patel (2010) reported that NSKE 5 per cent proved to be most effective followed by neemazole @ $2 \mathrm{ml} / 10$ litre against jassid on soybean.

Sutaria et al., (2010) concluded that thiamethoxam $0.05 \%$, acetamiprid $0.04 \%$ and imidacloprid $0.01 \%$ were most effective treatments to control the jassid in soybean. 
Int.J.Curr.Microbiol.App.Sci (2018) 7(8): 3834-3840

Table.1 Efficacy of different insecticides against jassid on soybean

\begin{tabular}{|c|c|c|c|c|c|c|c|c|c|}
\hline \multirow{3}{*}{$\begin{array}{l}\text { Sr. } \\
\text { No. }\end{array}$} & \multirow[t]{3}{*}{ Treatments } & \multirow{3}{*}{$\begin{array}{l}\text { Conc. } \\
(\%)\end{array}$} & \multicolumn{7}{|c|}{ Number of jassid/three leaves } \\
\hline & & & \multirow{2}{*}{$\begin{array}{l}\text { Before } \\
\text { Spray }\end{array}$} & \multicolumn{3}{|c|}{ First spray } & \multicolumn{3}{|c|}{ Second spray } \\
\hline & & & & $\begin{array}{c}3 \\
\text { DAS }\end{array}$ & 7 DAS & $\begin{array}{c}10 \\
\text { DAS }\end{array}$ & $\begin{array}{c}3 \\
\text { DAS }\end{array}$ & $\begin{array}{c}7 \\
\text { DAS }\end{array}$ & $\begin{array}{c}10 \\
\text { DAS }\end{array}$ \\
\hline 1 & $\begin{array}{l}\text { Thiamethoxam } \\
25 \text { WG }\end{array}$ & 0.008 & $\begin{array}{l}2.37^{*} \\
(5.12)\end{array}$ & $\begin{array}{l}1.54^{\mathrm{bc}} \\
(1.87)\end{array}$ & $\begin{array}{l}1.57^{\mathrm{bc}} \\
(1.95)\end{array}$ & $\begin{array}{l}1.61^{\mathrm{bc}} \\
(2.10)\end{array}$ & $\begin{array}{l}1.49^{\mathrm{bc}} \\
(1.71)\end{array}$ & $\begin{array}{l}1.53^{\mathrm{bcd}} \\
(1.84)\end{array}$ & $\begin{array}{l}1.56^{\mathrm{cd}} \\
(1.93)\end{array}$ \\
\hline 2 & $\begin{array}{l}\text { Buprofezin } 25 \\
\text { SC }\end{array}$ & 0.025 & $\begin{array}{c}2.34 \\
(4.98)\end{array}$ & $\begin{array}{l}1.57^{\mathrm{bc}} \\
(1.97)\end{array}$ & $\begin{array}{l}1.58^{\mathrm{bc}} \\
(2.01)\end{array}$ & $\begin{array}{l}1.67^{\mathrm{bcd}} \\
(2.27)\end{array}$ & $\begin{array}{l}1.52^{\mathrm{bcd}} \\
(1.82)\end{array}$ & $\begin{array}{l}1.55^{\mathrm{bcd}} \\
(1.90)\end{array}$ & $\begin{array}{l}1.59^{\mathrm{bcd}} \\
(2.04)\end{array}$ \\
\hline 3 & $\begin{array}{l}\text { Beauveria } \\
\text { bassiana } 2 \times 10^{8} \\
\text { cfu/g }\end{array}$ & 0.400 & $\begin{array}{c}2.41 \\
(5.30)\end{array}$ & $\begin{array}{l}1.89^{\mathrm{d}} \\
(3.06)\end{array}$ & $\begin{array}{l}1.90^{\mathrm{d}} \\
(3.11)\end{array}$ & $\begin{array}{l}1.91^{\mathrm{cd}} \\
(3.16)\end{array}$ & $\begin{array}{l}1.81^{\mathrm{cd}} \\
(2.76)\end{array}$ & $\begin{array}{l}1.85^{\mathrm{d}} \\
(2.93)\end{array}$ & $\begin{array}{l}1.87^{\mathrm{de}} \\
(2.98)\end{array}$ \\
\hline 4 & $\begin{array}{l}\text { Lecanicillium } \\
\text { lecanii } 2 \times 10^{8} \\
\text { cfu/g }\end{array}$ & 0.400 & $\begin{array}{c}2.33 \\
(4.91)\end{array}$ & $\begin{array}{l}1.90^{\mathrm{d}} \\
(3.12)\end{array}$ & $\begin{array}{l}1.92^{\mathrm{d}} \\
(3.17)\end{array}$ & $\begin{array}{l}1.95^{\mathrm{d}} \\
(3.29)\end{array}$ & $\begin{array}{l}1.84^{\mathrm{d}} \\
(2.90)\end{array}$ & $\begin{array}{l}1.87^{\mathrm{d}} \\
(3.01)\end{array}$ & $\begin{array}{l}1.90^{\mathrm{e}} \\
(3.11)\end{array}$ \\
\hline 5 & $\begin{array}{l}\text { Imidacloprid } \\
\text { 17.8 SL }\end{array}$ & 0.005 & $\begin{array}{c}2.30 \\
(4.78)\end{array}$ & $\begin{array}{l}1.24^{\mathrm{a}} \\
(1.03)\end{array}$ & $\begin{array}{l}1.27^{\mathrm{a}} \\
(1.11)\end{array}$ & $\begin{array}{l}1.32^{\mathrm{a}} \\
(1.24)\end{array}$ & $\begin{array}{l}1.15^{\mathrm{a}} \\
(0.82)\end{array}$ & $\begin{array}{l}1.15^{\mathrm{a}} \\
(0.83)\end{array}$ & $\begin{array}{c}1.27^{\mathrm{a}} \\
(1.12)\end{array}$ \\
\hline 6. & $\begin{array}{l}\text { Acetamiprid } 20 \\
\text { SP }\end{array}$ & 0.004 & $\begin{array}{c}2.31 \\
(4.84)\end{array}$ & $\begin{array}{l}1.36^{\mathrm{ab}} \\
(1.35)\end{array}$ & $\begin{array}{l}1.40^{\mathrm{ab}} \\
(1.45)\end{array}$ & $\begin{array}{l}1.43^{\mathrm{ab}} \\
(1.53)\end{array}$ & $\begin{array}{l}1.30^{\mathrm{ab}} \\
(1.20)\end{array}$ & $\begin{array}{l}1.34^{\mathrm{ab}} \\
(1.30)\end{array}$ & $\begin{array}{l}1.39^{\mathrm{ab}} \\
(1.44)\end{array}$ \\
\hline 7 & $\begin{array}{l}\text { Dimethoate } 30 \\
\text { EC }\end{array}$ & 0.030 & $\begin{array}{c}2.38 \\
(5.16)\end{array}$ & $\begin{array}{l}1.42^{\mathrm{ab}} \\
(1.51)\end{array}$ & $\begin{array}{l}1.47^{\mathrm{ab}} \\
(1.66)\end{array}$ & $\begin{array}{l}1.48^{\mathrm{ab}} \\
(1.70)\end{array}$ & $\begin{array}{l}1.33^{\mathrm{ab}} \\
(1.28)\end{array}$ & $\begin{array}{l}1.40^{\mathrm{abc}} \\
(1.47)\end{array}$ & $\begin{array}{l}1.46^{\mathrm{ab}} \\
(1.63)\end{array}$ \\
\hline 8 & $\begin{array}{l}\text { Neem oil } 1500 \\
\text { ppm }\end{array}$ & 0.500 & $\begin{array}{c}2.31 \\
(4.85)\end{array}$ & $\begin{array}{l}1.79^{\mathrm{cd}} \\
(2.69)\end{array}$ & $\begin{array}{l}1.83^{\mathrm{cd}} \\
(2.86)\end{array}$ & $\begin{array}{l}1.85^{\mathrm{cd}} \\
(2.91)\end{array}$ & $\begin{array}{l}1.68^{\mathrm{cd}} \\
(2.32)\end{array}$ & $\begin{array}{l}1.76^{\mathrm{bc}} \\
(2.58)\end{array}$ & $\begin{array}{l}1.79^{\text {cde }} \\
(2.72)\end{array}$ \\
\hline 9 & $\begin{array}{l}\text { Chlorfenapyr } 10 \\
\text { SC }\end{array}$ & 0.040 & $\begin{array}{c}2.27 \\
(4.64)\end{array}$ & $\begin{array}{l}1.62^{\mathrm{bcd}} \\
(2.11)\end{array}$ & $\begin{array}{l}1.62^{\mathrm{bcd}} \\
(2.13)\end{array}$ & $\begin{array}{l}1.70^{\text {bcd }} \\
(2.37)\end{array}$ & $\begin{array}{l}1.58^{\mathrm{bcd}} \\
(2.00)\end{array}$ & $\begin{array}{l}1.59^{\mathrm{bcd}} \\
(2.02)\end{array}$ & $\begin{array}{c}1.62^{\text {bcde }} \\
(2.12)\end{array}$ \\
\hline 10 & Control & - & $\begin{array}{c}2.31 \\
(4.85)\end{array}$ & $\begin{array}{c}2.33^{\mathrm{e}} \\
(4.94)\end{array}$ & $\begin{array}{l}2.38^{\mathrm{e}} \\
(5.15)\end{array}$ & $\begin{array}{l}2.40^{\mathrm{e}} \\
(5.25)\end{array}$ & $\begin{array}{l}2.44 \mathrm{e} \\
(5.45)\end{array}$ & $\begin{array}{l}2.54^{\mathrm{e}} \\
(5.93)\end{array}$ & $\begin{array}{c}2.67^{t} \\
(6.64)\end{array}$ \\
\hline & S.Em. \pm & & 0.13 & 0.09 & 0.09 & 0.09 & 0.10 & 0.11 & 0.09 \\
\hline & C.V. $\%$ & & 9.48 & 9.33 & 9.59 & 9.16 & 10.47 & 11.33 & 9.40 \\
\hline
\end{tabular}

* Figures outside parenthesis are $\sqrt{X+0.5}$ transformed values, while those in parenthesis are *retransformed value 
Int.J.Curr.Microbiol.App.Sci (2018) 7(8): 3834-3840

Table.2 Efficacy of different insecticides against whitefly on soybean

\begin{tabular}{|c|c|c|c|c|c|c|c|c|c|}
\hline \multirow{3}{*}{$\begin{array}{l}\text { Sr. } \\
\text { No. }\end{array}$} & \multirow[t]{3}{*}{ Treatments } & \multirow{3}{*}{$\begin{array}{c}\text { Conc. } \\
(\%)\end{array}$} & \multicolumn{7}{|c|}{ Number of whiteflies/three leaves } \\
\hline & & & \multirow{2}{*}{$\begin{array}{l}\text { Before } \\
\text { Spray }\end{array}$} & \multicolumn{3}{|c|}{ First spray } & \multicolumn{3}{|c|}{ Second spray } \\
\hline & & & & $\begin{array}{c}3 \\
\text { DAS }\end{array}$ & $\begin{array}{c}7 \\
\text { DAS }\end{array}$ & $\begin{array}{c}10 \\
\text { DAS }\end{array}$ & $\begin{array}{c}3 \\
\text { DAS }\end{array}$ & $\begin{array}{c}7 \\
\text { DAS }\end{array}$ & $\begin{array}{c}10 \\
\text { DAS }\end{array}$ \\
\hline 1. & $\begin{array}{l}\text { Thiamethoxam } \\
25 \mathrm{WG}\end{array}$ & 0.008 & $\begin{array}{l}2.59^{*} \\
(6.21)\end{array}$ & $\begin{array}{l}1.41^{\mathrm{ab}} \\
(1.50)\end{array}$ & $\begin{array}{l}1.45^{\mathrm{ab}} \\
(1.59)\end{array}$ & $\begin{array}{l}1.49^{\mathrm{ab}} \\
(1.72)\end{array}$ & $\begin{array}{l}1.36^{\mathrm{ab}} \\
(1.34)\end{array}$ & $\begin{array}{l}1.42^{\mathrm{ab}} \\
(1.51)\end{array}$ & $\begin{array}{l}1.47^{\mathrm{ab}} \\
(1.66)\end{array}$ \\
\hline 2. & $\begin{array}{l}\text { Buprofezin } 25 \\
\text { SC }\end{array}$ & 0.025 & $\begin{array}{c}2.62 \\
(6.35)\end{array}$ & $\begin{array}{l}1.76^{\mathrm{bc}} \\
(2.61)\end{array}$ & $\begin{array}{l}1.80^{\mathrm{abc}} \\
(2.73)\end{array}$ & $\begin{array}{l}1.82^{\mathrm{abc}} \\
(2.80)\end{array}$ & $\begin{array}{l}1.70^{\mathrm{bc}} \\
(2.38)\end{array}$ & $\begin{array}{l}1.77^{\mathrm{bc}} \\
(2.63)\end{array}$ & $\begin{array}{c}1.79^{\mathrm{abcd}} \\
(2.72)\end{array}$ \\
\hline 3. & $\begin{array}{l}\text { Beauveria } \\
\text { bassiana } 2 \times 10^{8} \\
\text { cfu/g }\end{array}$ & 0.400 & $\begin{array}{c}2.50 \\
(5.77)\end{array}$ & $\begin{array}{l}1.93^{\mathrm{c}} \\
(3.23)\end{array}$ & $\begin{array}{l}1.95^{\mathrm{c}} \\
(3.29)\end{array}$ & $\begin{array}{l}2.00^{\mathrm{c}} \\
(3.51)\end{array}$ & $\begin{array}{l}1.86^{\mathrm{c}} \\
(2.94)\end{array}$ & $\begin{array}{l}1.94^{\mathrm{c}} \\
(3.27)\end{array}$ & $\begin{array}{l}2.00^{\text {cd }} \\
(3.51)\end{array}$ \\
\hline 4. & $\begin{array}{l}\text { Lecanicillium } \\
\text { lecanii } 2 \times 10^{8} \\
\text { cfu/g }\end{array}$ & 0.400 & $\begin{array}{c}2.62 \\
(6.38)\end{array}$ & $\begin{array}{l}1.95^{\mathrm{c}} \\
(3.29)\end{array}$ & $\begin{array}{c}1.97^{\mathrm{c}} \\
(3.37)\end{array}$ & $\begin{array}{l}2.04^{\mathrm{c}} \\
(3.67)\end{array}$ & $\begin{array}{l}1.88^{\mathrm{c}} \\
(3.05)\end{array}$ & $\begin{array}{l}1.97^{\mathrm{c}} \\
(3.39)\end{array}$ & $\begin{array}{l}2.04^{\mathrm{d}} \\
(3.65)\end{array}$ \\
\hline 5. & $\begin{array}{l}\text { Imidacloprid } \\
\text { 17.8 SL }\end{array}$ & 0.005 & $\begin{array}{c}2.72 \\
(6.92)\end{array}$ & $\begin{array}{l}1.58^{\mathrm{abc}} \\
(2.01)\end{array}$ & $\begin{array}{l}1.61^{\mathrm{abc}} \\
(2.09)\end{array}$ & $\begin{array}{l}1.64^{\mathrm{abc}} \\
(2.19)\end{array}$ & $\begin{array}{l}1.50^{\mathrm{abc}} \\
(1.76)\end{array}$ & $\begin{array}{l}1.59^{\mathrm{abc}} \\
(2.03)\end{array}$ & $\begin{array}{l}1.62^{\mathrm{abc}} \\
(2.12)\end{array}$ \\
\hline 6. & $\begin{array}{l}\text { Acetamiprid } 20 \\
\text { SP }\end{array}$ & 0.004 & $\begin{array}{c}2.42 \\
(5.34)\end{array}$ & $\begin{array}{l}1.35^{\mathrm{a}} \\
(1.32)\end{array}$ & $\begin{array}{l}1.40^{\mathrm{a}} \\
(1.45)\end{array}$ & $\begin{array}{r}1.43^{\mathrm{a}} \\
(1.56)\end{array}$ & $\begin{array}{l}1.26^{\mathrm{a}} \\
(1.09)\end{array}$ & $\begin{array}{l}1.37^{\mathrm{a}} \\
(1.39)\end{array}$ & $\begin{array}{l}1.41^{\mathrm{a}} \\
(1.49)\end{array}$ \\
\hline 7. & $\begin{array}{l}\text { Dimethoate } 30 \\
\text { EC }\end{array}$ & 0.030 & $\begin{array}{c}2.62 \\
(6.38)\end{array}$ & $\begin{array}{l}1.75^{\mathrm{bc}} \\
(2.57)\end{array}$ & $\begin{array}{l}1.78^{\mathrm{abc}} \\
(2.67)\end{array}$ & $\begin{array}{l}1.80^{\mathrm{abc}} \\
(2.73)\end{array}$ & $\begin{array}{l}1.63^{\mathrm{abc}} \\
(2.14)\end{array}$ & $\begin{array}{l}1.75^{\mathrm{abc}} \\
(2.57)\end{array}$ & $\begin{array}{c}1.77^{\text {abcd }} \\
(2.63)\end{array}$ \\
\hline 8. & $\begin{array}{l}\text { Neem oil } 1500 \\
\text { ppm }\end{array}$ & 0.500 & $\begin{array}{c}2.45 \\
(5.50)\end{array}$ & $\begin{array}{l}1.85^{\mathrm{C}} \\
(2.94)\end{array}$ & $\begin{array}{l}1.88^{\mathrm{bc}} \\
(3.05)\end{array}$ & $\begin{array}{l}1.95^{\mathrm{c}} \\
(3.30)\end{array}$ & $\begin{array}{l}1.82^{\mathrm{c}} \\
(2.80)\end{array}$ & $\begin{array}{l}1.90^{\mathrm{c}} \\
(3.09)\end{array}$ & $\begin{array}{l}1.92^{\text {cd }} \\
(3.20)\end{array}$ \\
\hline 9. & $\begin{array}{l}\text { Chlorfenapyr } 10 \\
\text { SC }\end{array}$ & 0.040 & $\begin{array}{c}2.52 \\
(5.87)\end{array}$ & $\begin{array}{l}1.79^{\mathrm{bc}} \\
(2.72)\end{array}$ & $\begin{array}{l}1.81^{\mathrm{abc}} \\
(2.78)\end{array}$ & $\begin{array}{l}1.84^{\mathrm{bc}} \\
(2.88)\end{array}$ & $\begin{array}{l}1.70^{\mathrm{bc}} \\
(2.40)\end{array}$ & $\begin{array}{l}1.78^{\mathrm{bc}} \\
(2.66)\end{array}$ & $\begin{array}{l}1.81^{\mathrm{bcd}} \\
(2.78)\end{array}$ \\
\hline 10. & Control & - & $\begin{array}{c}2.40 \\
(5.24)\end{array}$ & $\begin{array}{l}2.41^{\mathrm{d}} \\
(5.29)\end{array}$ & $\begin{array}{l}2.46^{\mathrm{d}} \\
(5.53)\end{array}$ & $\begin{array}{l}2.49^{\mathrm{d}} \\
(5.71)\end{array}$ & $\begin{array}{l}2.56^{\mathrm{d}} \\
(6.07)\end{array}$ & $\begin{array}{l}2.61^{\mathrm{d}} \\
(6.31)\end{array}$ & $\begin{array}{l}2.63^{\mathrm{e}} \\
(6.40)\end{array}$ \\
\hline & S.Em. \pm & & 0.14 & 0.12 & 0.13 & 0.12 & 0.12 & 0.12 & 0.12 \\
\hline & C.V. \% & & 9.67 & 12.16 & 12 & 11.00 & 11.71 & 11.90 & 10.92 \\
\hline
\end{tabular}

* Figures outside parenthesis are $\sqrt{X+0.5}$ transformed values, while those in parenthesis are retransformed value. 
Int.J.Curr.Microbiol.App.Sci (2018) 7(8): 3834-3840

Table.3 Efficacy of different insecticides against thrips on soybean

\begin{tabular}{|c|c|c|c|c|c|c|c|c|c|}
\hline \multirow{3}{*}{$\begin{array}{l}\text { Sr. } \\
\text { No. }\end{array}$} & \multirow[t]{3}{*}{ Treatments } & \multirow{3}{*}{$\begin{array}{l}\text { Conc. } \\
(\%)\end{array}$} & \multicolumn{7}{|c|}{ Number of thrips/three leaves } \\
\hline & & & \multirow{2}{*}{$\begin{array}{l}\text { Before } \\
\text { Spray }\end{array}$} & \multicolumn{3}{|c|}{ First spray } & \multicolumn{3}{|c|}{ Second spray } \\
\hline & & & & 3 DAS & 7 DAS & 10 DAS & 3 DAS & 7 DAS & 10 DAS \\
\hline 1. & Thiamethoxam 25 WG & 0.008 & $\begin{array}{l}2.48 * \\
(5.66)\end{array}$ & $\begin{array}{l}1.38^{\mathrm{ab}} \\
(1.41)\end{array}$ & $\begin{array}{l}1.40^{\mathrm{ab}} \\
(1.46)\end{array}$ & $\begin{array}{l}1.52^{\mathrm{ab}} \\
(1.81)\end{array}$ & $\begin{array}{l}1.32^{\mathrm{ab}} \\
(1.24)\end{array}$ & $\begin{array}{l}1.38^{\mathrm{ab}} \\
(1.41)\end{array}$ & $\begin{array}{l}1.48^{\mathrm{ab}} \\
(1.70)\end{array}$ \\
\hline 2. & Buprofezin $25 \mathrm{SC}$ & 0.025 & $\begin{array}{c}2.52 \\
(5.84)\end{array}$ & $\begin{array}{l}1.69^{\text {bcd }} \\
(2.37)\end{array}$ & $\begin{array}{l}1.73^{\text {bcd }} \\
(2.49)\end{array}$ & $\begin{array}{l}1.79^{\mathrm{abc}} \\
(2.71)\end{array}$ & $\begin{array}{l}1.59^{\mathrm{bc}} \\
(2.01)\end{array}$ & $\begin{array}{l}1.67^{\text {bcd }} \\
(2.30)\end{array}$ & $\begin{array}{l}1.71^{\mathrm{bc}} \\
(2.43)\end{array}$ \\
\hline 3. & $\begin{array}{l}\text { Beauveria bassiana } 2 \times \\
10^{8} \mathrm{cfu} / \mathrm{g}\end{array}$ & 0.400 & $\begin{array}{c}2.50 \\
(5.75)\end{array}$ & $\begin{array}{l}1.92^{\text {cd }} \\
(3.18)\end{array}$ & $\begin{array}{l}1.95^{\mathrm{cd}} \\
(3.30)\end{array}$ & $\begin{array}{l}2.02^{\mathrm{c}} \\
(3.59)\end{array}$ & $\begin{array}{l}1.79^{c} \\
(2.69)\end{array}$ & $\begin{array}{l}1.88^{\text {cd }} \\
(3.05)\end{array}$ & $\begin{array}{l}1.94^{\mathrm{c}} \\
(3.27)\end{array}$ \\
\hline 4. & $\begin{array}{l}\text { Lecanicillium lecanii } 2 \\
\times 10^{8} \mathrm{cfu} / \mathrm{g}\end{array}$ & 0.400 & $\begin{array}{c}2.58 \\
(6.17)\end{array}$ & $\begin{array}{l}1.94^{\mathrm{d}} \\
(3.28)\end{array}$ & $\begin{array}{l}2.00^{\mathrm{d}} \\
(3.49)\end{array}$ & $\begin{array}{l}2.06^{\mathrm{cd}} \\
(3.75)\end{array}$ & $\begin{array}{l}1.83^{\mathrm{c}} \\
(2.84)\end{array}$ & $\begin{array}{l}1.94^{\mathrm{d}} \\
(3.25)\end{array}$ & $\begin{array}{l}1.97^{\mathrm{c}} \\
(3.39)\end{array}$ \\
\hline 5. & Imidacloprid $17.8 \mathrm{SL}$ & 0.005 & $\begin{array}{c}2.48 \\
(5.63)\end{array}$ & $\begin{array}{l}1.26^{\mathrm{a}} \\
(1.08)\end{array}$ & $\begin{array}{l}1.31^{\mathrm{a}} \\
(1.22)\end{array}$ & $\begin{array}{l}1.37^{\mathrm{a}} \\
(1.39)\end{array}$ & $\begin{array}{l}1.22^{\mathrm{a}} \\
(0.99)\end{array}$ & $\begin{array}{l}1.30^{\mathrm{a}} \\
(1.19)\end{array}$ & $\begin{array}{l}1.36^{\mathrm{a}} \\
(1.34)\end{array}$ \\
\hline 6. & Acetamiprid $20 \mathrm{SP}$ & 0.004 & $\begin{array}{c}2.56 \\
(6.04)\end{array}$ & $\begin{array}{l}1.52^{\mathrm{abc}} \\
(1.80)\end{array}$ & $\begin{array}{l}1.56^{\mathrm{abc}} \\
(1.93)\end{array}$ & $\begin{array}{l}1.57^{\mathrm{ab}} \\
(1.97)\end{array}$ & $\begin{array}{l}1.40^{\mathrm{ab}} \\
(1.47)\end{array}$ & $\begin{array}{l}1.53^{\mathrm{abc}} \\
(1.84)\end{array}$ & $\begin{array}{l}1.55^{\mathrm{ab}} \\
(1.91)\end{array}$ \\
\hline 7. & Dimethoate $30 \mathrm{EC}$ & 0.030 & $\begin{array}{c}2.42 \\
(5.38)\end{array}$ & $\begin{array}{l}1.65^{\text {bcd }} \\
(2.23)\end{array}$ & $\begin{array}{l}1.69^{\text {abcd }} \\
(2.36)\end{array}$ & $\begin{array}{l}1.77^{\mathrm{abc}} \\
(2.63)\end{array}$ & $\begin{array}{l}1.56^{\mathrm{bc}} \\
(1.95)\end{array}$ & $\begin{array}{l}1.66^{\text {bcd }} \\
(2.27)\end{array}$ & $\begin{array}{l}1.70^{\mathrm{bc}} \\
(2.38)\end{array}$ \\
\hline 8. & Neem oil 1500 ppm & 0.500 & $\begin{array}{c}2.69 \\
(6.23)\end{array}$ & $\begin{array}{l}1.88^{\mathrm{cd}} \\
(3.05)\end{array}$ & $\begin{array}{l}1.90^{\mathrm{cd}} \\
(3.12)\end{array}$ & $\begin{array}{l}1.95^{\mathrm{bc}} \\
(3.31)\end{array}$ & $\begin{array}{l}1.76^{\mathrm{C}} \\
(2.60)\end{array}$ & $\begin{array}{l}1.85^{\mathrm{cd}} \\
(2.93)\end{array}$ & $\begin{array}{c}1.90^{\mathrm{c}} \\
(3.09)\end{array}$ \\
\hline 9. & Chlorfenapyr 10 SC & 0.040 & $\begin{array}{c}2.52 \\
(5.86)\end{array}$ & $\begin{array}{l}1.71^{\text {bcd }} \\
(2.44)\end{array}$ & $\begin{array}{l}1.74^{\text {bcd }} \\
(2.54)\end{array}$ & $\begin{array}{l}1.81^{\mathrm{bc}} \\
(2.77)\end{array}$ & $\begin{array}{l}1.61^{b c} \\
(2.10)\end{array}$ & $\begin{array}{l}1.71^{\text {bcd }} \\
(2.42)\end{array}$ & $\begin{array}{l}1.74^{b c} \\
(2.53)\end{array}$ \\
\hline 10. & Control & - & $\begin{array}{c}2.34 \\
(4.97)\end{array}$ & $\begin{array}{l}2.36^{\mathrm{e}} \\
(5.05)\end{array}$ & $\begin{array}{l}2.41^{\mathrm{e}} \\
(5.31)\end{array}$ & $\begin{array}{l}2.50^{\mathrm{d}} \\
(5.77)\end{array}$ & $\begin{array}{c}2.53^{\mathrm{d}} \\
(5.90)\end{array}$ & $\begin{array}{l}2.57^{\mathrm{e}} \\
(6.09)\end{array}$ & $\begin{array}{l}2.60^{\mathrm{d}} \\
(6.25)\end{array}$ \\
\hline \multirow{2}{*}{\multicolumn{3}{|c|}{ S.Em. \pm}} & 0.14 & 0.12 & 0.12 & 0.13 & 0.09 & 0.11 & 0.10 \\
\hline & & & 9.70 & 12.00 & 11.99 & 12.01 & 9.65 & 11.30 & 10.00 \\
\hline
\end{tabular}

* Figures outside parenthesis are $\sqrt{X+0.5}$ transformed values, while those in parenthesis are retransformed value.

Table.4 Yield and avoidable losses in soybean treated with different insecticides

\begin{tabular}{|c|c|c|c|c|c|}
\hline $\begin{array}{l}\text { Sr. } \\
\text { No. }\end{array}$ & Treatments & $\begin{array}{c}\text { Concentration } \\
(\%)\end{array}$ & $\begin{array}{l}\text { Yield } \\
\text { (kg/ha) }\end{array}$ & $\begin{array}{l}\text { Increased in yield } \\
\text { over control }(\%)\end{array}$ & $\begin{array}{l}\text { Avoidable } \\
\text { loss }(\%)\end{array}$ \\
\hline 1 & Thiamethoxam $25 \mathrm{WG}$ & 0.008 & $1092^{\mathrm{ab}}$ & 38.05 & 06.34 \\
\hline 2 & Buprofezin 25 SC & 0.025 & $943^{\mathrm{cd}}$ & 19.21 & 19.12 \\
\hline 3 & $\begin{array}{l}\text { Beauveria bassiana } 2 \times \\
10^{8} \mathrm{cfu} / \mathrm{g}\end{array}$ & 0.400 & $876^{\text {cde }}$ & 10.74 & 24.87 \\
\hline 4 & $\begin{array}{l}\text { Lecanicillium lecanii } 2 \\
\times 10^{8} \mathrm{cfu} / \mathrm{g}\end{array}$ & 0.400 & $865^{\text {de }}$ & 09.35 & 25.81 \\
\hline 5 & Imidacloprid 17.8 SL & 0.005 & $1166^{\mathrm{a}}$ & 47.40 & 00.00 \\
\hline 6 & Acetamiprid $20 \mathrm{SP}$ & 0.004 & $1114^{\mathrm{ab}}$ & 40.83 & 04.45 \\
\hline 7 & Dimethoate $30 \mathrm{EC}$ & 0.030 & $1012^{\mathrm{bc}}$ & 27.93 & 13.20 \\
\hline 8 & Neem oil 1500 ppm & 0.500 & $897^{\text {cde }}$ & 13.40 & 23.07 \\
\hline 9 & Chlorfenapyr 10 SC & 0.040 & $930^{\mathrm{cd}}$ & 17.57 & 20.24 \\
\hline 10 & Control & - & $791^{\mathrm{e}}$ & - & 32.16 \\
\hline \multicolumn{2}{|c|}{ S.Em. \pm} & 42.10 & - & - & - \\
\hline \multicolumn{2}{|c|}{ C.V. \% } & 07.53 & - & - & - \\
\hline
\end{tabular}




\section{Whitefly (B. tabaci)}

The results on incidence of whitefly before and after spraying are summarised in Table 2 . Based on the results of first and second spray, it was evident that the acetamiprid 20 SP @ $0.004 \%$ proved the most effective treatment in controlling whitefly under field conditions on soybean followed by thiamethoxam 25 WG@ $0.008 \%$ and imidacloprid 17.8 SL @ $0.005 \%$. The treatment comprising buprofezin 25 SC @ $0.025 \%$, dimethoate 30 EC @ $0.03 \%$ and chlorfenapyr 10 SC @ 0.04 $\%$ formed the next effective group of insecticides against whitefly. Similarly, nonchemical insecticides viz., neem oil $1500 \mathrm{ppm}$ @ $0.5 \%$, Beauveria bassiana @ $2 \times 10^{8} \mathrm{cfu} / \mathrm{g}$ and Lecanicillium lecanii $2 \times 10^{8} \mathrm{cfu} / \mathrm{g}$ had also proved their superiority against untreated control. Looking to the eco-friendly approach, these bio-pesticides can be incorporated in IPM programme against whitefly.

Pande et al., (2008) stated that NSKE $5 \%$ considerably reduced the population of whitefly on soybean. Patel et al., (2009) reported that the imidacloprid $17.8 \mathrm{SL}$ was significantly superior in reducing the whitefly population in clusterbean.

\section{Thrips (S. dorsalis)}

The results on effect of different treatments on incidence of thrips before and after spraying are summarised in Table 3. Based on first and second sprays of the insecticides, it can be summarised that the imidacloprid 17.8 SL@ $0.005 \%$ was the most effective treatment in controlling thrips of soybean under field conditions followed by thiamethoxam 25 WG @ $0.008 \%$ and acetamiprid 20 SP @ $0.004 \%$. Similarly non chemical insecticides viz., neem oil $1500 \mathrm{ppm}$ @ $0.5 \%$, Beauveria bassiana $2 \times 10^{8} \mathrm{cfu} / \mathrm{g}$ and Lecanicillium lecanii $2 \times 10^{8} \mathrm{cfu} / \mathrm{g}$ also found effective against untreated control and can be incorporated in IPM programme against thrips. Earlier, Dahiphale et al., (2007) and Abbaszadeh (2014) reported imidacloprid as effective insecticides against sucking pest of soybean. Imidacloprid 17.8 SL, thiamethoxam $25 \mathrm{WG}$ and acetamiprid 20 SP were found effective against the sucking pest of clusterbean, moth bean and mung bean as reported by Patel et al., (2009).

\section{Yield}

The results on yield increase in yield over control and avoidable losses are presented in Table 4. The highest yield of soybean was recorded in the treatment of imidacloprid 17.8 SL@ $0.005 \%(1166 \mathrm{~kg} / \mathrm{ha})$ and was at par with acetamiprid 20 SP @ $0.004 \%$ (1114 $\mathrm{kg} / \mathrm{ha}$ ) and thiamethoxam 25 WG @ $0.008 \%$ $(1092 \mathrm{~kg} / \mathrm{ha})$. The treatments of dimethoate 30 EC @ $0.03 \%$ (1012 kg/ha), buprofezin 25 SC @ $0.025 \%$ (943 kg/ha) and chlorfenapyr 10 SC @ $0.04 \%$ (930 kg/ha) formed the next group of effective treatments where, the yields ranged between 930 and $1092 \mathrm{~kg} / \mathrm{ha}$. All the non-chemical treatments viz., Beauveria bassiana $2 \times 10^{8} \mathrm{cfu} / \mathrm{g}$, neem oil 1500 ppm @ $0.5 \%$ and Lecanicillium lecanii $2 \times 10^{8} \mathrm{cfu} / \mathrm{g}$ could not perform significantly over control in respect to yield of soybean which ranged between 865 and $897 \mathrm{~kg} / \mathrm{ha}$.

\section{Increased in yield over control}

Per cent increase in soybean yield over control due to various treatments was worked out and presented in Table 4.

Results showed that the per cent increase in yield over control was maximum in the treatment of imidacloprid 17.8 SL @ $0.005 \%$ $(47.40 \%)$ and it was followed by acetamiprid 20 SP @ $0.004 \%$ (40.83\%), thiamethoxam 25 WG @ $0.008 \%$ (38.05\%) and dimethoate 30 EC@ $0.03 \%$ (27.93 \%). However, the lowest increase in yield over control was 
obtained in the treatment of Lecanicillium lecanii $2 \times 10^{8} \mathrm{cfu} / \mathrm{g}(9.35 \%)$.

\section{Avoidable losses}

Percentage of avoidable losses in soybean yield due to sucking pests (jassid, whitefly and thrips) after applying various treatments was worked out by applying formula suggested by Khosla (1977) and presented in Table 4. It can be seen from the results that the maximum grain yield was obtained in the treatment of imidacloprid 17.8 SL @ $0.005 \%$ which proved as the best treatment. The avoidable loss in soybean due to sucking pests varied from 4.45 to $32.16 \%$. The avoidable losses in soybean yield was minimum in the plot treated with acetamiprid 20 SP @ 0.004 $\%(4.45 \%)$ followed by thiamethoxam 25 WG @ $0.008 \%$ (6.34 \%). On other hand. The highest percentage of avoidable loss in soybean yield was observed in untreated plots $(32.16 \%)$.

\section{References}

Abbaszadeh, G. (2014). Efficacy of different insecticides against dubas bug, Ommatissus lybicus. Annals of Plant Protection Sciences. 22: 240-243.

Ahirwar, K.C.; Das, S.B.; Bhowmick, A.K. and Gupta, M.P. (2015). Succession of insect pests and their natural enemies on soybean (Glycine max L.). Annals of Plant Protection Sciences. 23 (1): 5254.

Biswas, G.C. (2013). Insect pests of soybean (Glycine $\max$ L.), their nature of damage and succession with the crop stages. Journal of the Asiatic Society of Bangladesh, Science. 39 (1): 1-8.

Dahiphale, K.D., Suryawanshi, D.S., Kamble, S.K. and Pole, S.P. (2007). Effect of new insecticides against the control of major insect pests and yield of soybean [Glycine $\max$ (L.) Merrill]. Soybean Research. 5 (7): 87-90.

Joshi, M.D. and Patel, V.N. (2010). Efficacy of Eco-friendly insecticides against jassid (Empoasca kerri P.). Legume Research. 33 (3): 231-232.

Khosla, R.K. (1977). Techniques for assessment of losses due to pests and diseases of rice. Indian Journal of Agricultural Science. 47(4): 171-174.

Lal, S.S., Yadav, C.P. and Dias, C.A. (1981). Insect pests of pulse crops and their management. Pesticide. 21: 66-67.

Pande, R., Firake, D.M. and Yadav, S. (2008). Study of neem seed kernel extract with different additives, synthetic and biopesticides against soybean whitefly. Journal of Eco-friendly Agriculture. 3 (2): $168-170$

Patel, P.S., Patel, I.S., Panickar, B. and Acharya, S. (2009). Evaluation of newer insecticides against sucking insect pests of cluster bean. International Conference on Nurturing Arid Zones for People and the Environment: Issues and Agenda for $21^{\text {st }}$ Century. Arid Zone Research Association of India, CAZRI Campus, Jodhpur, India. p. 102.

Sutaria, V.K., Motka, M.N., Jethva, D.M. and Ramoliya, D.R. (2010). Field efficacy of insecticides against Jassid, Empoasca kerri (Pruthi) in Soybean. Annals of Plant Protection Science. 18 (1): 94-97.

\section{How to cite this article:}

Dinesh M. Chaudhary, Mahesh M. Chaudhary and Chaudhary, F.K. 2018. Evaluation of Newer Insecticidal Formulation against Sucking Pests and Effect on Yield of Soybean (Glycine max L.). Int.J.Curr.Microbiol.App.Sci. 7(08): 3834-3840. doi: https://doi.org/10.20546/ijcmas.2018.708.392 